\title{
PERTURBING ASYMPTOTICALLY STABLE DIFFERENTIAL EQUATIONS
}

\author{
BY AARON STRAUSS ${ }^{1}$ AND JAMES A. YORKE ${ }^{2}$
}

Communicated by W. Wasow, April 22, 1968

Our purpose here is to announce new theorems on the eventual uniform-asymptotic stability (hereafter called EvUAS) of the origin 0 for the ordinary differential equation

$$
x^{\prime}=f(t, x)+g(t, x), \quad \quad\left(x^{\prime}=d x / d t\right)
$$

given that 0 is EvUAS for the equation

$$
x^{\prime}=f(t, x),
$$

and that $f$ and $g$ satisfy certain conditions. We always assume that $f$ and $g$ are at least continuous from $[0, \infty) \times R^{d}$ to $R^{d}$, but we never assume that the solutions of $(\mathrm{P})$ are unique or that the zero function is a solution of $(\mathrm{P})$. In fact EvUAS is a natural generalization of uniform asymptotic stability in which it is not assumed that the zero function is a solution.

Our main result is (definitions follow)

Theorem A. Let 0 be EvUAS for (E). Then 0 is EvUAS for (P) if

(i) $f$ is Lipschitz and $g$ is diminishing, or

(ii) $f$ is periodic and $g$ is diminishing, or

(iii) $f$ is inner product and $g$ is absolutely diminishing, or

(iv) $f$ is linear and $g=g_{1}+g_{2}$, where $g_{1}$ is absolutely diminishing and $g_{2}=o(|x|)$.

Let $x\left(t ; t_{0}, x_{0}\right)$ denote a solution of $(\mathrm{E})$ through $\left(t_{0}, x_{0}\right)$. We say that 0 is EvUAS for (E) if

$$
\lim _{t 0 \rightarrow \infty ;\left|x_{0}\right| \rightarrow 0}\left[\sup _{t \geq t_{0}}\left|x\left(t ; t_{0}, x_{0}\right)\right|\right]=0
$$

and if, for some $\delta_{0}>0$ and some $\alpha_{0} \geqq 0$,

$$
\lim _{t \rightarrow \infty}\left[\sup _{t_{0} \geqq \alpha_{0} ;\left|x_{0}\right|<\delta_{0}}\left|x\left(t+t_{0} ; t_{0}, x_{0}\right)\right|\right]=0 .
$$

1 Department of Mathematics, University of Maryland, College Park, Maryland. Research supported in part by a National Science Foundation Postdoctoral Fellowship and in part by NSF grants GP-4921 and GP-6167.

2 Institute for Fluid Dynamics and Applied Mathematics, University of Maryland, College Park, Maryland. Research supported in part by NSF grants GP-6114 and GP-7846. 
We say that $f$ is Lipschitz if, for some $r>0$ and $L>0$, $|f(t, x)-f(t, y)| \leqq L|x-y|$ for $t \geqq 0,|x| \leqq r$, and $|y| \leqq r ;$ inner product if, for some $r>0$ and $L>0$,

$$
\langle x-y, f(t, x)-f(t, y)\rangle \leqq L\|x-y\|^{2} \text { for } t \geqq 0,|x| \leqq r, \text { and }|y| \leqq r ;
$$

linear if

$$
f(t, x)=A(t) x \quad \text { for } t \geqq 0 \text { and } \quad x \in R^{d} ;
$$

and periodic if, for some $\omega>0$,

$$
f(t+\omega, x)=f(t, x) \text { for } t \geqq 0 \text { and } x \in R^{d} .
$$

Note that if $A(t)$ is bounded on $[0, \infty)$, then $f(t, x)=A(t) x$ is both linear and Lipschitz; that $f$ is periodic if it is independent of $t$; and that a Lipschitz function is also inner product.

We say that $g$ is absolutely diminishing if, for some $r>0$ and every $m$ satisfying $0<m<r$, there exists a function $h_{m}$ such that, for all $t \geqq 0$ and $m \leqq|x| \leqq r$,

$$
|g(t, x)| \leqq h_{m}(t) \text { and } \int_{t}^{t+1} h_{m}(s) d s \rightarrow 0 \quad \text { as } t \rightarrow \infty \text {. }
$$

We say that $g$ is diminishing if: (i) $g$ is absolutely diminishing; or (ii) $g$ is continuous in $x$ uniformly with respect to $t \in[0, \infty)$ and, for some $r>0$ and each fixed $x$ satisfying $0<|x|<r$,

$$
\sup _{0 \leqq u \leqq 1}\left|\int_{t}^{t+u} g(s, x) d s\right| \rightarrow 0 \quad \text { as } t \rightarrow \infty ;
$$

or (iii) $g$ is a finite sum of functions of types (i) and (ii).

Theorem A generalizes the following result, obtained in stages by Malkin [3, p. 104], Vrkoc [7], Wexler [8], Yoshizawa [9], Krasovskii [4, p. 102], LaSalle and Rath [5], and Strauss and Yorke [6]:

THEOREM. If 0 is UAS for (E), if $f$ is Lipschitz, and if $g$ is absolutely diminishing, then 0 is EvUAS for (P).

Theorem A also generalizes the following result, obtained in stages by Poincaré, Liapunov, Perron, Coddington and Levinson [2, p. 327], Brauer [1], and Strauss and Yorke [6]:

TheOREM. Let $A$ be a constant matrix. If 0 is $U A S$ for $x^{\prime}=A x$ and if $g=g_{1}+g_{2}$, where $g_{1}$ is absolutely diminishing (but with $m=0$ ) and $g_{2}=o(|x|)$, then 0 is "eventually asymptotically stable" for $(\mathrm{P})$. 
There do not seem to be any results in the literature for $f$ merely periodic or inner product,

We now briefly discuss diminishing functions. If $|g(t, x)| \leqq h(t)$ for all $t \geqq 0$ and $|x| \leqq r$, then $g$ is absolutely diminishing whenever, in particular, $h(t) \rightarrow 0$ as $t \rightarrow \infty$ or

$$
\int_{0}^{\infty}|h(t)|^{p} d t<\infty \quad \text { for some } p \geqq 1 .
$$

The scalar function $g(t, x)=t\left(t^{2} x^{2}+1\right)^{-1}$ is absolutely diminishing because we may choose

$$
h_{m}(t)=t\left(t^{2} m^{2}+1\right)^{-1} \rightarrow 0 \text { as } t \rightarrow \infty ;
$$

however, $g(t, 0)=t$. Thus an absolutely diminishing function need not be small at $x=0$. The function

$$
h(t)=\left(t \sin t^{3}, t \cos t^{8}, 0, \cdots, 0\right)
$$

is diminishing but not absolutely diminishing, since $\|h(t)\|=t$. Furthermore, $t^{-1} h(t)$ is bounded and diminishing, but not absolutely diminishing. If $k(x)$ is continuous from $R^{d}$ to $R^{d}$ (we need not have $k(0)=0$ ), then the function $k(x) \sin t^{3}$ is diminishing but not absolutely diminishing. Examples show that if uniform continuity is dropped from the definition of a diminishing function, then part (i) of Theorem A may fail.

We now summarize some of our other results.

Theorem B. Let 0 be EvUAS for (E). Let $f$ be Lipschitz or periodic. Then 0 is EvUAS for

$$
x^{\prime}=f(t, x)+h(t)
$$

if and only if $h$ is diminishing. In fact if $h$ is not diminishing, then no solution of (1) can approach zero as $t \rightarrow \infty$.

Both implications of Theorem B are false tor inner product $f$ and for linear $f$. Furthermore, there exist diminishing functions $g$ and there exist functions $f$ which are both inner product and linear such that 0 is EvUAS for (E) but not for $(\mathrm{P})$.

To understand better the relationship between properties of $f$ in (E) and the conditions for admissible perturbations $g$, we use the concept of perturbation classes. Define

$$
\Im_{\mathrm{C}}=\left\{f(t, x): f \text { is continuous from }[0, \infty) \times R^{d} \text { to } R^{d}\right\} .
$$


Let $\mathfrak{F}$ be a subclass of $\mathfrak{F}_{\mathrm{C}}$. Define the perturbation classes

$$
\begin{aligned}
\mathscr{S}(F) & =\left\{g \in \mathscr{F}_{\mathrm{C}}: \forall f \in \mathscr{F}, 0 \text { is EvUAS for }(\mathrm{E}) \Rightarrow 0 \text { is EvUAS for }(\mathrm{P})\right\}, \\
\mathfrak{F C}(F) & =\{h \in \mathcal{G}(\mathfrak{F}): h \text { is independent of } x\} .
\end{aligned}
$$

Then if $\mathfrak{F}_{\text {Lip }}$ denotes the class of Lipschitz functions, $\mathfrak{F}_{I n n}$ the class of inner product functions, $\mathfrak{F}_{L \text { in }}$ the class of linear functions, and $\mathcal{F}_{\mathbf{P}_{\text {er }}}$ the class of periodic functions, we may restate Theorem $\mathrm{A}$ as

$$
\begin{aligned}
& \mathcal{G}\left(F_{\text {Lip }}\right) \supset\{g(t, x): g \text { is diminishing }\}, \\
& \mathcal{G}\left(\mathcal{F}_{\text {Per }}\right) \supset\{g(t, x): g \text { is diminishing }\}, \\
& \mathcal{G}\left(\mathcal{F}_{\text {Inn }}\right) \supset\{g(t, x): g \text { is absolutely diminishing }\}, \\
& \mathcal{G}\left(F_{\text {Lin }}\right) \supset\left\{g_{1}+g_{2}: g_{1} \text { is absolutely diminishing and } g_{2}=o(|x|)\right\} .
\end{aligned}
$$

Theorem B implies

$$
\mathcal{H C}\left(\mathcal{F}_{\text {Lip }}\right)=\mathcal{H C}\left(\mathcal{F}_{\text {Per }}\right)=\{h(t): h \text { is diminishing }\} .
$$

The remarks following Theorem $B$ imply $F_{C}\left(F_{L i p}\right) \neq F_{C}\left(F_{L i n}\right)$ and $\mathfrak{H C}\left(\mathcal{F}_{\text {Lip }}\right) \neq \mathfrak{F C}\left(\mathcal{F}_{\text {Inn }}\right)$.

The conditions we impose on $g$ are that $g$ be "small as $t \rightarrow \infty$." We can use conditions of the type " $g$ is small as $|x| \rightarrow 0$ " and still perturb every equation (E) for $f$ linear, but not for $f$ Lipschitz, inner product, or periodic, as Theorem $\mathrm{A}$ and the next result show.

TheOREM C. Let $d \geqq 2$. Then we have the following:

for Lipschitz $g(x), g \in \mathcal{G}\left(\mathcal{F}_{\text {Lip }}\right) \Leftrightarrow g(x) \equiv 0$ near $x=0$;

for Lipschitz $g(x), g \in \mathcal{G}\left(\mathcal{F}_{\text {Inn }}\right) \Leftrightarrow g(x) \equiv 0$ near $x=0$;

for continuous $g(x), g \in \mathcal{G}\left(F_{\text {Per }}\right) \Leftrightarrow g(x) \equiv 0$ near $x=0$;

for a constant matrix $A, A x \in \mathcal{G}\left(F_{\text {Lin }}\right) \Leftrightarrow A x=\alpha x$ for some $\alpha \leqq 0$.

Finally, we show that restrictions on $f$ (such as Lipschitz, etc.) are needed in order to prove a result like Theorem A. Let $F_{\mathrm{CU}}$ be the class of functions which are locally Lipschitz and uniformly continuous on $[0, \infty) \times R^{d}$.

THEOREM D. For some $f \in \mathcal{F}_{\mathrm{CU}}, 0$ is EvUAS for (E) but not for

$$
x^{\prime}=f(t, x)+e^{-t}(1, \cdots, 1) .
$$

Also, for some $f \in F_{\mathrm{CU}}, 0$ is $E v U A S$ for $(\mathrm{E})$ but not for

$$
x^{\prime}=f(t, x)+x e^{-t} \text {. }
$$

In particular, then, $e^{-t}(1, \cdots, 1) \notin \mathcal{G}\left(F_{\mathrm{CU}}\right)$ and $x e^{-t} \notin \mathcal{G}\left(F_{\mathrm{CU}}\right)$. 


\section{REFERENCES}

1. F. Brauer, Nonlinear differential equations with forcing terms, Proc. Amer. Math. Soc. 15 (1964), 758-765.

2. E. A. Coddington and N. Levinson, Theory of ordinary differential equations, McGraw-Hill, New York, 1955.

3. A. Halanay, Differential equations, Academic Press, New York and London, 1966.

4. N. Krasovskii, Stability of motion, Stanford Univ. Press, Stanford, California, 1963.

5. J. P. LaSalle and R. J. Rath, Eventual stability, Proc. 2nd IFAC Congress, Basel, 1963, Butterworth, London, 1964, Vol. II, pp. 556-560.

6. A. Strauss and J. A. Yorke, Perturbation theorems for ordinary differential equations, J. Differential Equations 3 (1967), 15-30.

7. I. Vrkoc, Integral stability, Czech. Math. J. 9 (1959), 71-128. (Russian)

8. D. Wexler, Note on the eventual stability, Rev, Roum. Math. Pures et Appl. 11 (1966), 819-824.

9. T. Yoshizawa, Stability theory by Liapunov's second method, Math. Soc. Japan, Tokyo, 1966.

UNIVERSITY OF MARYLAND 